\title{
Neutrino wave function and oscillation suppression.
}

\author{
A.D. Dolgov ${ }^{* a, b}$, O.V. Lychkovskiy, ${ }^{\dagger c}$, A.A. Mamonov ${ }^{\ddagger c}$, \\ L.B. Okun ${ }^{\S a}$, and M.G. Schepkin ${ }^{\text {Ia }}$ \\ a Institute of Theoretical and Experimental Physics \\ 117218, B.Cheremushkinskaya 25, Moscow, Russia \\ b INFN, Ferrara 40100, Italy \\ c Moscow Physics and Technology Institute
}

\begin{abstract}
We consider a thought experiment, in which a neutrino is produced by an electron on a nucleus in a crystal. The wave function of the oscillating neutrino is calculated assuming that the electron is described by a wave packet. If the electron is relativistic and the spatial size of its wave packet is much larger than the size of the crystal cell, then the wave packet of the produced neutrino has essentially the same size as the wave packet of the electron. We investigate the suppression of neutrino oscillations at large distances caused by two mechanisms: 1) spatial separation of wave packets corresponding to different neutrino masses; 2) neutrino energy dispersion for given neutrino mass eigenstates. We resolve contributions of these two mechanisms.
\end{abstract}

\section{Introduction.}

There are two different approaches to neutrino oscillations in the literature: one of them deals with the wave function of free neutrinos, while the other considers the propagator of virtual neutrinos. The latter approach was analyzed in the papers [1, 2] for a thought experiment, where a neutrino was considered to be produced by an electron on the target nucleus $A$, and captured by the nucleus $B$ in the detector. The process was described as a two-stage Feynman amplitude, $e+A \rightarrow C+\nu, \nu+B \rightarrow D+l$ with a virtual neutrino, its Green function connecting the production and detection points: $x_{A}=\left(t_{A}, \mathbf{x}_{A}\right)$ and $x_{B}=$ $\left(t_{B}, \mathbf{x}_{B}\right)$. As it is well known, at a large distance $r_{A B}=\left|\mathbf{x}_{B}-\mathbf{x}_{A}\right|$ from the production point virtual particles become effectively real and one may speak about their wave function, in

*e-mail: dolgov@itep.ru

†e-mail: lychkovskiy@mail.ru

${ }^{\ddagger}$ e-mail: mamonov@dgap.mipt.ru

$\S$ e-mail: okun@itep.ru

๑e-mail: schepkin@itep.ru 
particular, about the neutrino wave function, $\psi_{\nu}(x)$. Though this statement is well known and practically evident, an explicit expression for $\psi_{\nu}$ can be instructive for the description of the effect of oscillation suppression in a thought experiment.

There are two mechanisms of erasing oscillations. The first one is spatial separation of neutrino wave packets of different mass eigenstates (see refs. [3] - [6]). The second mechanism is caused by neutrino energy dispersion (see e.g. ref.[7]). The amplitude of the two-stage process $e+A \rightarrow C+\nu_{i}$ and $\nu_{i}+B \rightarrow D+l$ with a virtual neutrino of given mass $m_{i}$ is determined by the standard rules of quantum field theory [1]:

$$
A_{i}=\int d^{4} x_{1} d^{4} x_{2} \psi_{l}^{*}\left(x_{2}\right) \psi_{D}^{*}\left(x_{2}\right) \psi_{B}\left(x_{2}\right) G_{i}\left(x_{2}-x_{1}\right) \psi_{C}^{*}\left(x_{1}\right) \psi_{e}\left(x_{1}\right) \psi_{A}\left(x_{1}\right)
$$

where $x_{1,2}$ are 4-dimensional coordinates, $x_{k}=\left(t_{k}, \mathbf{x}_{k}\right), G_{i}\left(x_{2}-x_{1}\right)$ is the Green function of the $i$-th neutrino mass eigenstate, and non-essential spin factors are neglected. The term $P_{i j}=A_{i} A_{j}^{*}$ in the probability was integrated over the phase space of the final particles in ref.[1]. After integration the interference term with $i \neq j$ vanishes at large spatial separation $\left|\mathbf{x}_{A}-\mathbf{x}_{B}\right|$.

In this approach, however, we were unable to resolve the contributions of the two mechanisms. In this note we try to separate the "Siamese twins". For this purpose we consider only the first stage of the process, $e+A \rightarrow C+\nu$, with a free neutrino. We consider the case when the spatial size of the electron wave packet is much larger than the size of the crystal cell, which determines the localization of the nucleus. The opposite case will be described elsewhere.

\section{Wave function vs amplitude}

The non-normalized wave function of a neutrino produced in the reaction $e+A \rightarrow C+\nu$ is

$$
\begin{gathered}
\psi_{\nu}=\sum_{i} U_{e i} \psi_{i}\left|\nu_{i}\right\rangle \\
\psi_{i}(t, \mathbf{x})=\int d^{4} x_{1} G_{i}\left(x-x_{1}\right) \psi_{C}^{*}\left(x_{1}\right) \psi_{e}\left(x_{1}\right) \psi_{A}\left(x_{1}\right),
\end{gathered}
$$

where $x \equiv(t, \mathbf{x})$ is the space-time coordinate and $\left|\nu_{i}\right\rangle$ is the $i$-th neutrino mass eigenstate.

In this equation the product $\psi_{C}^{*}\left(x_{1}\right) \psi_{e}\left(x_{1}\right) \psi_{A}\left(x_{1}\right)$ serves as a local source of neutrinos. To calculate the amplitude of neutrino interaction with the nucleus $B$ one would evidently substitute this expression for $\psi_{i}$ into the integral over $x$ with the wave functions of other particles participating in the reaction, according to eq.(11). This naturally agrees with the general prescription.

Like in our recent paper [1] we consider the initial nucleus bound in a crystal and describe it by a stationary wave function localized near the point $\mathbf{x}=0$ :

$$
\Psi_{A}(x)=F_{A}(\mathbf{x}) e^{-i t E_{A}},
$$


where $E_{A}$ is the energy of the nucleus. The Fourier transform of $F_{A}(\mathbf{x})$, which is required in what follows, is

$$
K_{A}\left(\mathbf{q}_{A}\right)=\int d \mathbf{x} F_{A}(\mathbf{x}) e^{-i \mathbf{q}_{A} \mathbf{x}}
$$

By assumption, the nucleus $A$ is at rest and, thus, $K_{A}\left(\mathbf{q}_{A}\right)$ is centered at $\mathbf{q}_{A}=0$ with the uncertainty $\sigma_{A} \sim a^{-1}$.

The wave function of the incident electron is considered to be a wave packet:

$$
\Psi_{e}(x)=\int d \mathbf{q}_{e} K_{e}\left(\mathbf{q}_{e}-\mathbf{p}_{e}\right) e^{i \mathbf{q}_{e}\left(\mathbf{x}-\mathbf{x}_{e}\right)-i E_{e}\left(\mathbf{q}_{e}\right) t}=e^{i \mathbf{p}_{e}\left(\mathbf{x}-\mathbf{x}_{e}\right)-i E_{e}\left(\mathbf{p}_{e}\right) t} F_{e}\left(\mathbf{x}-\mathbf{e} v_{e} t\right) .
$$

Here $\mathbf{e} \equiv \mathbf{p}_{e} / p_{e}, p_{e} \equiv\left|\mathbf{p}_{e}\right|, E_{e}\left(\mathbf{q}_{e}\right) \equiv \sqrt{\mathbf{q}_{e}^{2}+m_{e}^{2}}$, the Fourier amplitude $K_{e}\left(\mathbf{q}_{e}-\mathbf{p}_{e}\right)$ is centered near $\mathbf{q}_{e}=\mathbf{p}_{e}$ with the uncertainty $\sigma_{e}$, the center of the packet envelope $F_{e}\left(\mathbf{x}-\mathbf{e} v_{e} t\right)$ is at the point $\mathbf{x}_{e}$ at the moment $t=0$, and the electron group velocity $v_{e}$ is defined as

$$
\left.v_{e} \equiv \frac{\partial E_{e}\left(\mathbf{q}_{e}\right)}{\partial q_{e}}\right|_{\mathbf{q}_{e}=\mathbf{p}_{e}}=\frac{p_{e}}{\sqrt{p_{e}^{2}+m_{e}^{2}}} \simeq 1-\frac{m_{e}^{2}}{2 p_{e}^{2}} .
$$

In what follows we assume the electron to hit the nucleus $A$ at $t=0$ and the collision to be central, i.e. $\mathbf{x}_{e}=0$.

The recoil nucleus $C$ is described by the plane wave

$$
\Psi_{C}^{*}(x)=e^{i t E_{C}-i \mathbf{p}_{C} \mathbf{x}}
$$

unless its momentum is comparable with $\sigma_{A}$, which is an extremely rare case.

For the Green function the following expression can be derived

$$
G_{i}(t, \mathbf{x})=-\frac{1}{4 \pi|\mathbf{x}|} \int_{-\infty}^{\infty} d \omega e^{-i \omega t+i \sqrt{\omega^{2}-m_{i}^{2}}|\mathbf{x}|}
$$

Let us now substitute expressions (31), (15), (17) and (18) into eq.(21) and perform trivial integration over $x_{1}$ :

$$
\begin{gathered}
\psi_{i}(t, \mathbf{x})=\frac{1}{r} \int d \omega d \mathbf{q}_{e} K_{e}\left(\mathbf{q}_{e}-\mathbf{p}_{e}\right) d \mathbf{q}_{A} K_{A}\left(\mathbf{q}_{A}\right) \\
\delta\left(E_{e}\left(\mathbf{q}_{e}\right)+E_{A}-E_{C}-\omega\right) \delta\left(\mathbf{q}_{e}+\mathbf{q}_{A}-\mathbf{p}_{C}-\mathbf{k}_{i}\right) \exp \left(i k_{i} r-i \omega t\right) .
\end{gathered}
$$

Here $r \equiv|\mathbf{x}|, \mathbf{k}_{i} \equiv \mathbf{n} k_{i}, \mathbf{n} \equiv \mathbf{x} / r, k_{i} \equiv \sqrt{\omega^{2}-m_{i}^{2}}$ and the expansion

$$
\left|\mathbf{x}-\mathbf{x}_{1}\right| \approx r-\mathbf{n} \mathbf{x}_{1}
$$

is performed assuming $r$ to be much larger than the interaction region. In eq.(92) and in what follows we omit some non-essential numerical factors. We integrate eq.(9) over $\mathbf{q}_{A}$ and $\omega$ and obtain

$$
\psi_{i}(x)=\frac{1}{r} \int d \mathbf{q}_{e} K_{e}\left(\mathbf{q}_{e}-\mathbf{p}_{e}\right) K_{A}\left(-\mathbf{q}_{e}+\mathbf{p}_{C}+\mathbf{k}_{i}\right) \exp \left[i k_{i} r-i \omega t\right],
$$


where now $\omega\left(\mathbf{q}_{e}\right) \equiv E_{e}\left(\mathbf{q}_{e}\right)+E_{A}-E_{C}$.

While proceeding with the calculations we bear in mind the range and hierarchy of the quantities involved:

$$
m_{i} \ll \sigma_{e} \ll \sigma_{A} \ll m_{e} \ll p_{e} \ll M_{A}, M_{C} .
$$

In particular, we consider the case when $K_{A}\left(\mathbf{q}_{A}\right)$ is wide in comparison with $K_{e}\left(\mathbf{q}_{e}\right)$ :

$$
\sigma_{e} \ll \sigma_{A} .
$$

This allows to set $K_{A}\left(-\mathbf{q}_{e}+\mathbf{p}_{C}+\mathbf{k}_{i}\right)=K_{A}\left(-\mathbf{p}_{e}+\mathbf{p}_{C}+\mathbf{k}_{i}^{0}\right),\left.\mathbf{k}_{i}^{0} \equiv \mathbf{k}_{i}\right|_{\mathbf{q}_{e}=\mathbf{p}_{e}}$ throughout the essential range of integration over $\mathbf{q}_{e}$. Assuming that the momentum distribution of the electron is sufficiently narrow we may expand the integrand in terms of $\mathbf{q}_{e}$ near the central electron momentum $\mathbf{p}_{e}$ :

$$
\begin{array}{r}
\omega\left(\mathbf{q}_{e}\right)=\omega^{0}+v_{e} \mathbf{e}\left(\mathbf{q}_{e}-\mathbf{p}_{e}\right), \\
k_{i}\left(\mathbf{q}_{e}\right)=k_{i}^{0}+\frac{v_{e}}{v_{i}} \mathbf{e}\left(\mathbf{q}_{e}-\mathbf{p}_{e}\right),
\end{array}
$$

where we introduce the neutrino group velocities analogously to the electron one:

$$
v_{i} \equiv\left(\frac{\partial \omega}{\partial k_{i}}\right)^{0}=\frac{\sqrt{\left(\omega^{0}\right)^{2}-m_{i}^{2}}}{\omega^{0}} \simeq 1-\frac{m_{i}^{2}}{2\left(\omega^{0}\right)^{2}} ;
$$

the upper index "0" means that the corresponding quantities are calculated at $\mathbf{q}_{e}=\mathbf{p}_{e}$.

Taking into account eqs.(15), (11) and (14) we obtain the following simple expression for the wave packet of the produced neutrino through the envelope of the wave packet of the incoming electron in coordinate space, $F_{e}$ :

$$
\psi_{i}(t, r, \mathbf{n})=\frac{e^{i k_{i}^{0} r-i \omega^{0} t}}{r} K_{A}\left(-\mathbf{p}_{e}+\mathbf{p}_{C}+k_{i}^{0} \mathbf{n}\right) F_{e}\left(\frac{v_{e}}{v_{i}}\left(r-v_{i} t\right) \mathbf{e}\right)
$$

The factor $v_{e} / v_{i} \simeq 1$ makes the neutrino wave packet a little bit wider then the electron one. It is not essential for our purposes and will be omitted in what follows.

Equation (16), which is one of the main results of this paper, is quite natural. If there were a long wave packet of the incoming electron, it would create a packet of neutrinos with a similar length. A good analogy is the scattering of a sound wave on a target which creates another sound wave. The duration and, correspondingly, the spatial length of the produced wave packet should be equal to the duration and size of the original one.

Strictly speaking, one has to calculate the amplitude (11) to determine the probability of the oscillating behavior of neutrinos. However, one may rely on a simplified approach based on the interpretation of the absolute value squared of the neutrino wave function as the probability density for the particle to be found at a spatial point $\mathbf{x}$ at a given time $t$. Such an approach is valid, if we deal with wave packets, the longitudinal size of which is much larger then their wave length, and their transversal size is much larger than their Compton wave length (see, e.g., [8, 9]). If we neither register the nucleus $C$ nor 
measure the time of neutrino detection, we are interested in the detection probability of the neutrino $\nu_{l}$ at point $\mathbf{x}$ :

$$
P_{\nu_{e} \rightarrow \nu_{l}}(\mathbf{x})=\int d \mathbf{p}_{C} d t\left|\sum_{i} U_{l i}^{*} U_{e i} \psi_{i}(t, \mathbf{x})\right|^{2} .
$$

For simplicity, in what follows we assume $i=1,2, l=e, \mu, U_{e 1}=\cos \theta, U_{e 2}=\sin \theta$. For the $\nu_{\mu}$ production probability we obtain

$$
\begin{gathered}
P_{\nu_{e} \rightarrow \nu_{\mu}}(\mathbf{x})=\frac{f}{2 r^{2}}(\sin 2 \theta)^{2} \int d \mathbf{p}_{C} K_{A}^{2}\left(-\mathbf{p}_{e}+\mathbf{p}_{C}+k_{i}^{0} \mathbf{n}\right) \\
{\left[1-\frac{1}{f} \cos \left(\frac{r \delta m^{2}}{2 \omega^{0}}\right) \int d t F_{e}\left(\left(r-v_{1} t\right) \mathbf{e}\right) F_{e}\left(\left(r-v_{2} t\right) \mathbf{e}\right)\right],}
\end{gathered}
$$

where $f \equiv \int d t F_{e}^{2}((r-t) \mathbf{e}), \delta m^{2} \equiv m_{2}^{2}-m_{1}^{2}$.

The term proportional to $\cos \left(r \delta m^{2} / 2 \omega^{0}\right)$ in eq.(18) describes oscillations. It vanishes at large distances for two different reasons.

\section{Packet separation.}

When $\left(v_{2}-v_{1}\right) r>1 / \sigma_{e}$, that is

$$
r>L_{o s c} \frac{p_{e}}{\sigma_{e}}, \quad L_{o s c} \equiv \frac{2 p_{e}}{\delta m^{2}}
$$

the product $F_{e}\left(r-v_{1} t\right) F_{e}\left(r-v_{2} t\right)$ is nearly zero for every $t$, and the oscillating term vanishes even before integration over $\mathbf{p}_{C}$.

\section{Neutrino energy dispersion.}

Note that $\omega^{0}$ depends on $\mathbf{p}_{C}$ :

$$
\omega^{0} \simeq E_{e}\left(\mathbf{p}_{e}\right)+E_{A}-M_{C}-\frac{p_{C}^{2}}{2 M_{C}}
$$

where $M$ denotes the $C$ nucleus mass. Owing to the factor $K_{A}^{2}\left(-\mathbf{p}_{e}+\mathbf{p}_{C}+k_{i}^{0} \mathbf{n}\right)$ in eq.(18), the effective size of the region of integration over $\mathbf{p}_{C}$ is of the order of $\sigma_{A}$. Thus $\omega^{0}$ varies near its central value (which is roughly equal to $p_{e}$ ), and the variation equals $p_{C}^{0} \sigma_{A} / M_{C}$. Here, the central value $\mathbf{p}_{C}^{0}$ is determined by the equation

$$
\mathbf{p}_{C}^{0}=\mathbf{p}_{e}-\left(p_{e}+\frac{m_{e}^{2}}{2 p_{e}}+E_{A}-M_{C}-\frac{\left(p_{C}^{0}\right)^{2}}{2 M_{C}}\right) \mathbf{n} .
$$

If the angle between vectors $\mathbf{e}$ and $\mathbf{n}$ is sufficiently large $\left(|\mathbf{e}-\mathbf{n}| p_{e} \gg M_{C}-E_{A}, p_{e}^{2} / 2 M_{C}\right)$, then

$$
p_{C}^{0} \simeq p_{e}|\mathbf{e}-\mathbf{n}|
$$

This is the case considered below. The variation of $\omega^{0}$ equals $p_{e}|\mathbf{e}-\mathbf{n}| \sigma_{A} / M_{C}$. Thus integration of $\cos \left(r \delta m^{2} / 2 \omega^{0}\right)$ over $\mathbf{p}_{C}$ in eq.(18) leads to the vanishing result if

$$
r>L_{o s c} \frac{M_{C}}{|\mathbf{e}-\mathbf{n}| \sigma_{A}} .
$$


We see that there are two competitive mechanisms for suppression of neutrino oscillations. If

$$
\sigma_{e}<\sigma_{A} \frac{p_{e}|\mathbf{e}-\mathbf{n}|}{M_{C}}
$$

then the energy dispersion mechanism dominates, and

$$
L_{\text {sup }}=L_{o s c} \frac{M_{C}}{|\mathbf{e}-\mathbf{n}| \sigma_{A}} .
$$

If

$$
\sigma_{A} \frac{p_{e}|\mathbf{e}-\mathbf{n}|}{M_{C}}<\sigma_{e} \ll \sigma_{A},
$$

then the packet separation works, and

$$
L_{\text {sup }}=L_{o s c} \frac{p_{e}}{\sigma_{e}} .
$$

These results coincide with those obtained in ref. 1 .

One more comment is worth making at this stage. As we said above, the interference disappears when two neutrino wave packets $\psi_{i}$ and $\psi_{j}$, eq.(16), cease to overlap. At first sight this statement is at odds with the expression for the production amplitude (11) of lepton $l$ on the nucleus $B$. Indeed, the product of amplitudes $A_{i}$ and $A_{j}^{*}$, which enters the probability of the process (see eqs.(27)-(29) of ref. 1]), does not vanish even when the product $\psi_{i}(x) \psi_{j}^{*}(x)$ vanishes, because the amplitude contains an integral over $x$ and the product of integrals never vanishes. However, one can check that after integration over the phase space of the final particles the product of the integrals vanishes exactly when the neutrino wave packets no longer overlap. Such integration over the phase space makes the result effectively local.

This conclusion is intuitively clear for the following reasons. The product of the amplitudes prior to integration over the phase space describes the production probability of a plane wave final state, because the final states are taken as momentum eigenfunctions. It is evident that such a probability never vanishes even in the case of infinite separation of neutrino wave packets. It is essentially the same as the excitation of a resonator by two wave packets. A resonator with a very large $Q$-factor would stop to oscillate only after a very long time. So, if such a resonator is hit by one wave packet and after a while by another delayed wave packet, the interference between the packets would still be observed by such a resonator because it keeps oscillating long after the fist packet has gone while the second has just arrived.

\section{Conclusions}

In this note we have considered neutrinos produced in the reaction $e+A \rightarrow C+\nu$. For the case of a large size of the electron wave packet $\left(\sigma_{e} \ll \sigma_{A}\right)$ we have calculated the neutrino wave packet (see eq.(16) ). Its size coincides with that of the incident electron wave packet.

We have demonstrated that in the case of $\sigma_{e}<p_{e}|\mathbf{e}-\mathbf{n}| \sigma_{A} / M_{C}$ the suppression of neutrino oscillations at large distances is due to the neutrino energy dispersion, while in 
the case of $p_{e}|\mathbf{e}-\mathbf{n}| \sigma_{A} / M_{C}<\sigma_{e} \ll \sigma_{A}$ it occurs because of the neutrino packet separation. The corresponding suppression lengths are given by eqs.(25) and (27). It is evident that for terrestrial conditions such lengths are unrealistic.

\section{Acknowledgments}

We are grateful to G.B. Pontecorvo and M.V. Rotaev for valuable discussions.

This work was partially supported by Dynasty Foundation, by the grant SH-2328.2003.2 and by the RFBR grant 05-02-17471.

\section{References}

[1] A.D. Dolgov, O.V. Lychkovskiy, A.A. Mamonov, L.B. Okun, M.V. Rotaev, M.G. Schepkin. hep-ph/0505251.

[2] I.Yu. Kobzarev, B.V. Martemyanov, L.B. Okun, M.G. Schepkin, Sov. J. Nucl. Phys. 35 (1982) 708.

[3] S. Nussinov, Phys. Lett. B63 (1976) 201.

[4] B. Kayser, Phys. Rev. D24 (1981) 110.

[5] A.D. Dolgov, Yad. Fiz. 33 (1981) 1309 [English translation Sov. J. Nucl. Phys. 33 (1981) 700].

[6] A.D. Dolgov, Neutrino Oscillations in Cosmology. A lecture presented at the 7th Course: Current Topics of Astrofundamental Physics ed. N. Sanchez, Erice-Sicile, 5-16 December, 1999 p. 565-584; hep-ph/0004032.

[7] D.E. Groom in Review of Particle Physics, Phys. Lett. B592 (2004) 451.

[8] J.D. Bjorken, S.D. Drell. "Relativistic Quantum Mechanics", v.1, McGraw-Hill Book Company., 1964.

[9] S. Weinberg. "The Quantum Theory of Fields", v.1, Cambridge University Press, 1995. 